\title{
The Sausage Technique using Collagen Membrane without Autogenous Bone Graft: A Case Report
}

\author{
Sung-Jo Lee, DDS, $\mathrm{PhD}^{1 *}$ \\ 'Assistant Professor, Department of Periodontology, Sejong Dental Hospital, Dankook University College of \\ Dentistry, Sejong-si, Korea \\ *Corresponding author: Sung-Jo Lee, Department of Periodontology, Sejong Dental Hospital, Dankook \\ University College of Dentistry, 87, Doum 8-ro, Sejong-si 30107, Korea. \\ Tel:+82-44-410-5051. Fax : +82-303-3442-7364. E-mail : periolee@dankook.ac.kr
}

\begin{abstract}
Various techniques have been introduced for the guided bone regeneration procedure. Among these, the sausage technique using a natural collagen membrane has many advantages. This report describes two cases wherein the sausage technique was employed in the maxillary anterior area using deproteinized bovine bone mineral (DBBM) and native bilayer collagen membrane (NBCM) without autogenous bone graft. In both cases, sufficient horizontal bone regeneration for implant placement was achieved. In addition, the bone and implant conditions were maintained without any complications maintained for more than 15 months. Within the limitations of this report, the sausage technique using NBCM with DBBM was able to achieve satisfactory horizontal alveolar bone augmentation for implant placement in the maxillary anterior area.
\end{abstract}

Keywords: Alveolar process, Bone regeneration, Dental implant, Space maintenance

pISSN 2765-7833

eISSN 2765-7841

Journal of implantology and applied sciences 2021; 25(2): 74-83

https://doi.org/10.32542/implantology.2021008

Received: March 2, 2021

Revised: April 22, 2021

Accepted: May 28, 2021

ORCID

Sung-Jo Lee

https://orcid.org/0000-0002-7834-209X

Copyright $\odot$ 2021. The Korean Academy of Oral \& Maxillofacial Implantology

This is an Open Access article distributed under the terms of the Creative Commons Attribution Non-Commercial License (http://creativecommons. org/licenses/by-nc/4.0/) which permits unrestricted non-commercial use, distribution, and reproduction in any medium, provided the original work is properly cited.

\section{I . Introduction}

A sufficient horizontal alveolar ridge width at an appropriate location is essential to meet the functional and esthetic demands of a dental implant. However, in some cases, it is not possible to place the implant in an ideal position due to insufficient alveolar bone width. In such cases, guided bone regeneration (GBR) should be considered. Various techniques have been introduced to increase the horizontal width of the alveolar bone. Among these, the sausage technique introduced by Istvan Urban was reported to enable successful bone regeneration by immobilizing the collagen membrane with a titanium pin and pushing the bone graft material in the crestal direction. ${ }^{1}$

Briefly, the technique is similar to conventional GBR in that the membrane is fixed with pins. However, the graft material is filled inside the fixed membrane in a sufficient quantity to show a balloon effect and to push the graft material in the crestal direction to create 
tension on the membrane. In this technique, two types of collagen membranes can be used: a crosslinked synthetic collagen membrane or a native collagen membrane. The author followed the technique of using a native collagen membrane. When a cross-linked synthetic collagen membrane is exposed, approximately $48.5 \%$ of the grafted bone may be lost. ${ }^{2}$ An inflammatory reaction may also occur during membrane degradation. ${ }^{3}$ On the other hand, due to rapid degradation of a natural collagen membrane, epithelialization proceeds rapidly in case of exposure and the risk of infection is relatively low. ${ }^{4,5} \mathrm{~A}$ cross-linked synthetic membrane does not allow capillaries to pass through it, but a natural collagen membrane is vascularized within the first weeks of healing. ${ }^{3}$ These advantages can reduce patient discomfort and the surgeon's burden in terms of complications.

It is known that resorbable membranes are less commonly used for GBR than non-resorbable membranes. ${ }^{6}$ However, according to the PASS (primary closure, angiogenesis, space maintenance, stability of wound) principle for successful GBR reported by Wang et al., the sausage technique is theoretically considered a predictable procedure, as it meets most of the principles. ${ }^{7}$ Stability of the wound and space maintenance can be enhanced by a combination of titanium pins, bone grafts, and membranes. The balloon effect of the membrane can be achieved by using a sufficient amount of bone graft with a slow resorption rate and pin fixation. ${ }^{1}$ Therefore, the author decided to proceed with the sausage technique while focusing on these advantages.

The present report describes the results of the sausage technique using native bilayer collagen membrane (NBCM) without autogenous bone graft in patients with moderate to severe alveolar bone resorption in the maxillary anterior area.

\section{II . Case Report}

\section{Case 1}

A 51-year-old healthy male patient presented to the Department of Periodontology at Dankook University Dental Hospital with mobility of the maxillary incisors and premolars. Clinical examination revealed grade 3 mobility with severe pain in the maxillary right premolars and grade 2 mobility in the incisors. Radiographic examination confirmed vertical and horizontal alveolar bone resorption around the maxillary right premolars and vertical alveolar bone resorption around the incisors (Fig. 1).

Tooth extraction and implant placement were prioritized to relieve pain in the right maxillary premolars. The maxillary incisors had a 4-month healing period after extraction and implant placement was planned for both lateral incisors (Fig. 2). At the request of the patient, implant placement was performed for the right and left lateral incisors at different times and the sausage technique was used for the right lateral incisor. The patient was instructed to rinse with $0.12 \%$ chlorhexidine digluconate solution (hexamedine, 


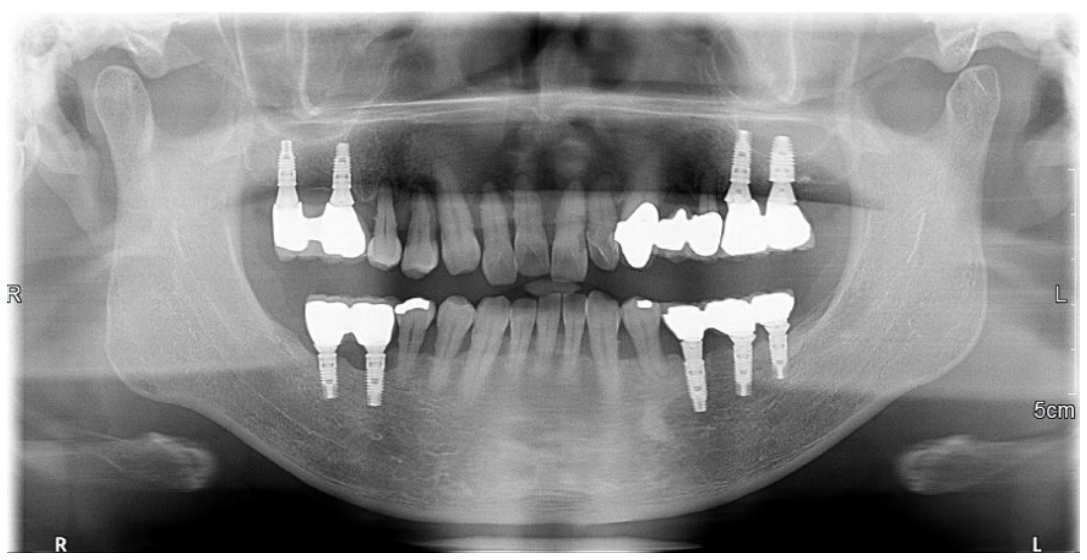

Fig. 1. Case 1: Panoramic radiograph at the first visit.

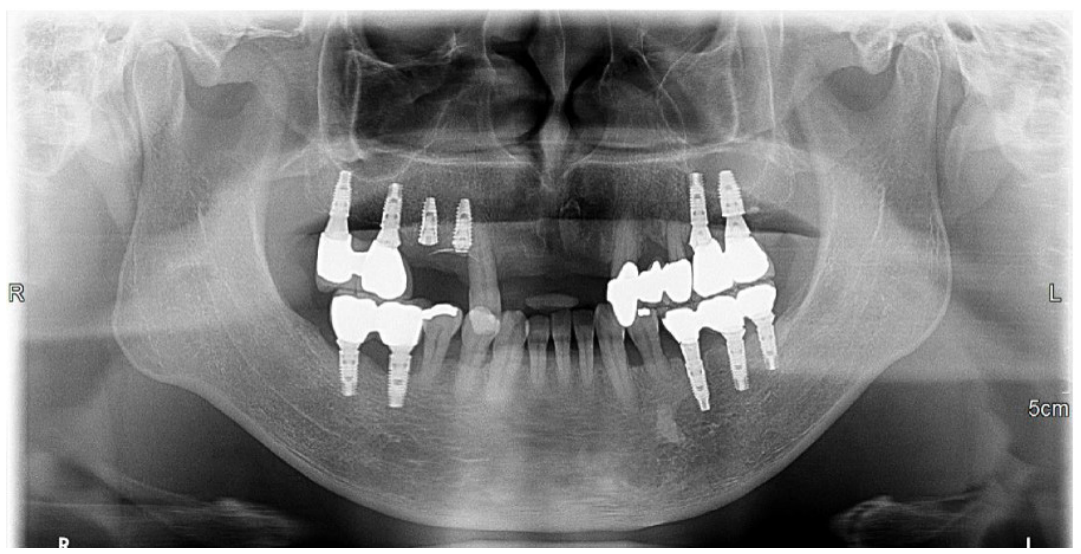

Fig. 2. Case 1: Panoramic radiograph at 4 months after extraction of the maxillary anterior teeth.

Bukwang, Seoul, Korea) for 2 minutes for oral disinfection. A crestal incision and two vertical releasing incisions were made under local anesthesia with $2 \%$ lidocaine containing 1:80,000 epinephrine (Huons Lidocaine, Huons, Seoul, Korea). The remaining alveolar ridge exhibited a height of $10 \mathrm{~mm}$ and a horizontal width of $5 \mathrm{~mm}$ after elevation of the flap (Fig. 3A). Deproteinized bovine bone mineral (DBBM) (Bio-Oss ${ }^{\circledR}$, Geistlich Pharma, Wolhusen, Switzerland) was placed in the defect. Subsequently, NBCM (Bio-Gide ${ }^{\circledR}$, Geistlich Pharma, Wolhusen, Switzerland) was fixed to the apical area of the remaining alveolar ridge using titanium pins with a head diameter of $3.2 \mathrm{~mm}$, pin diameter of $0.55 \mathrm{~mm}$, and a length of $2.8 \mathrm{~mm}$ (Membrane Pin, Dentium, Seoul, Korea) (Fig. 3B, 3C). The fixed membrane was stretched enough to produce a balloon effect and fixed with titanium pins to the crestal bone (Fig. 3D, 3E). The flap was sutured with 4-0 non-resorbable nylon monofilament (4-0 Ethilon; Ethicon, Cincinnati, OH, United States) using modified Laurell sutures and simple interrupted sutures (Fig. 3F).

After reopening for implant placement, the site demonstrated a horizontal bone width of $9 \mathrm{~mm}$ after 4 months of healing (Fig. 4A). Implant placement (IS III active ${ }^{\circledR}[4.0 \times 8.5 \mathrm{~mm}]$, NeoBiotech, Seoul, 
Korea) was performed without additional GBR and suturing was done with 4-0 non-resorbable nylon monofilament sutures (Fig. 4B, 4C). The Implant stability quotient (ISQ) value at implant placement was 69 . The second surgery was performed 4 months later. The bone and implant conditions were maintained without any complications for 15 months after placement of the prosthesis (Fig. 5A, 5B).
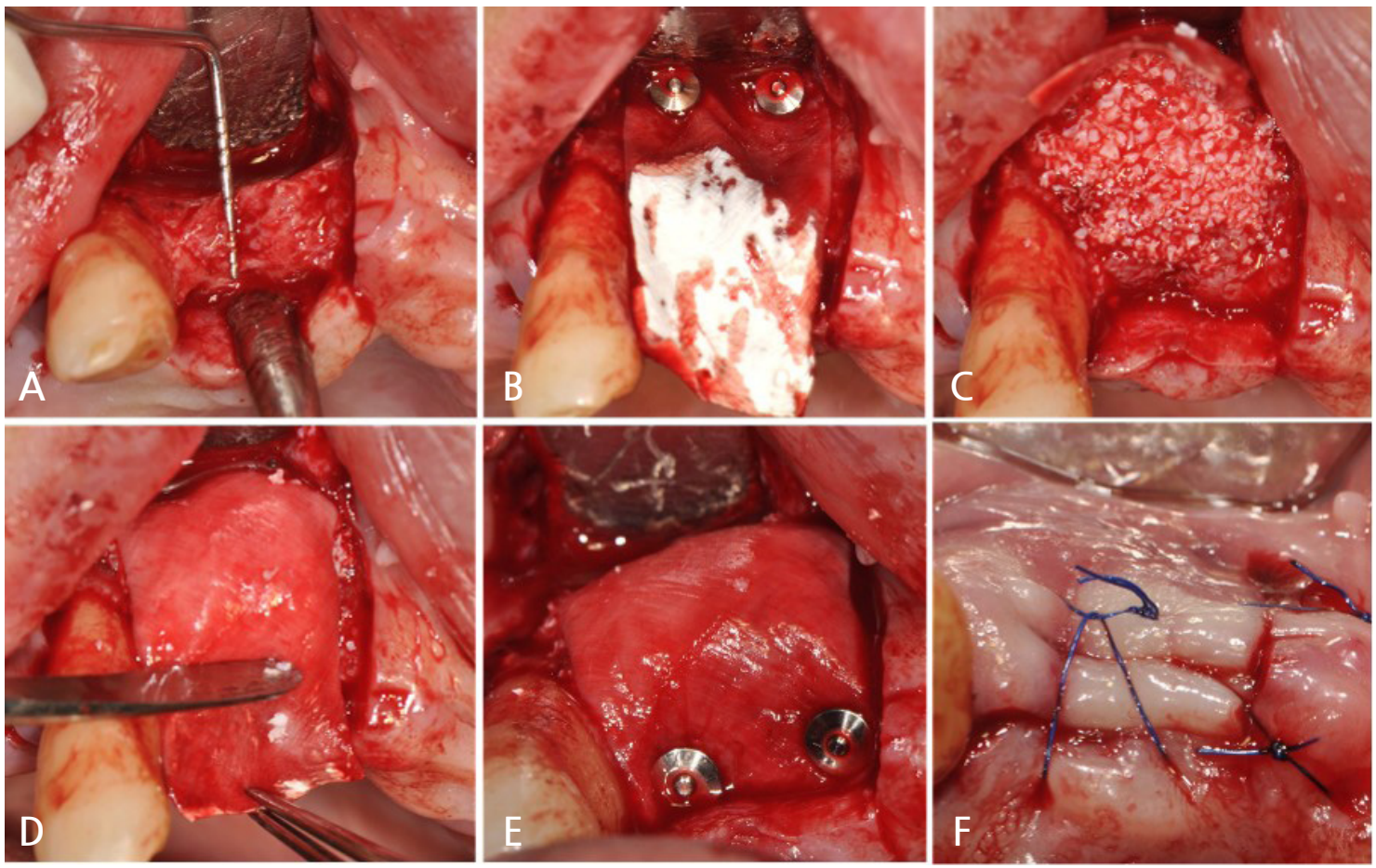

Fig. 3. Case 1: Clinical photographs of the guided bone regeneration procedure. (A) Horizontal alveolar ridge width of $5 \mathrm{~mm}$ was observed after flap elevation, (B) Native bilayer collagen membrane was fixed to the apical area of the remaining alveolar ridge using titanium pins, (C) Deproteinized bovine bone mineral was placed in the defect, (D) The fixed membrane was stretched upward for adequate stability, (E) The stretched collagen membrane was fixed to the crestal bone with titanium pins, (F) Suturing was performed using modified Laurell sutures at the crestal incision area and simple interrupted sutures at the vertical incision area.
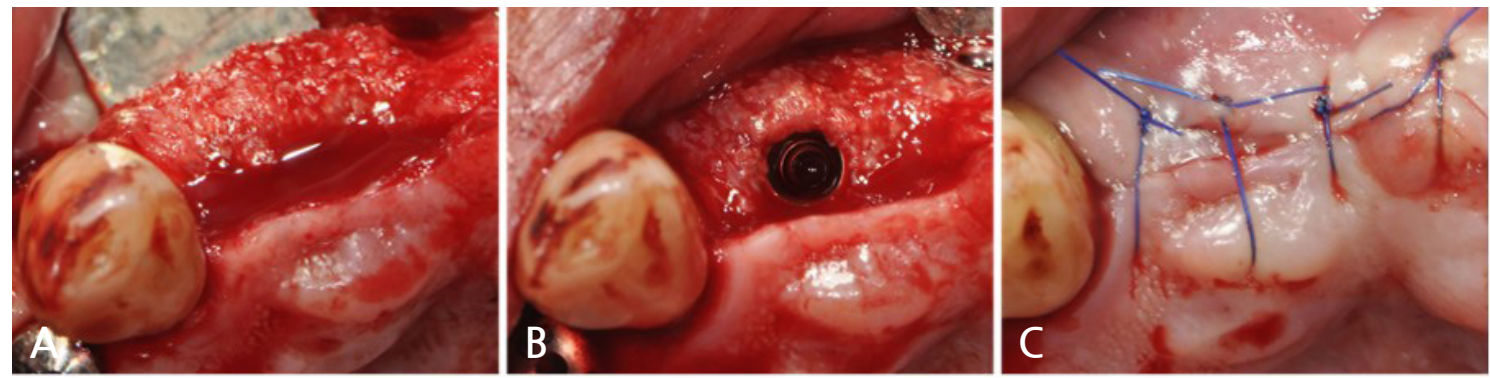

Fig. 4. Case 1: Clinical photographs of implant placement. (A) Horizontally augmented alveolar bone was observed after buccal flap elevation, (B) Implant placement was performed without additional guided bone regeneration, (C) The flap was secured using simple interrupted sutures. 


\section{Case 2}

A 64-year-old healthy male patient presented to the Department of Periodontology at Dankook University Dental Hospital with mobility of the maxillary incisors. Clinical examination revealed grade 3 mobility of the maxillary right lateral incisor, incisor, and left lateral incisor tooth. Vertical alveolar bone resorption around the incisors was confirmed by radiographic examination (Fig. 6). A 4-month healing period was observed after extraction of the maxillary incisors and the sausage technique was planned before implant placement (Fig. 7A, 7B). The patient was instructed to rinse with $0.12 \%$ chlorhexidine digluconate solution for 2 minutes before surgery. One crestal incision and two vertical releasing incisions were made under local anesthesia with $2 \%$ lidocaine containing 1:80,000 epinephrine. A two-wall defect in the right lateral incisor area and a horizontally reduced alveolar bone in the left

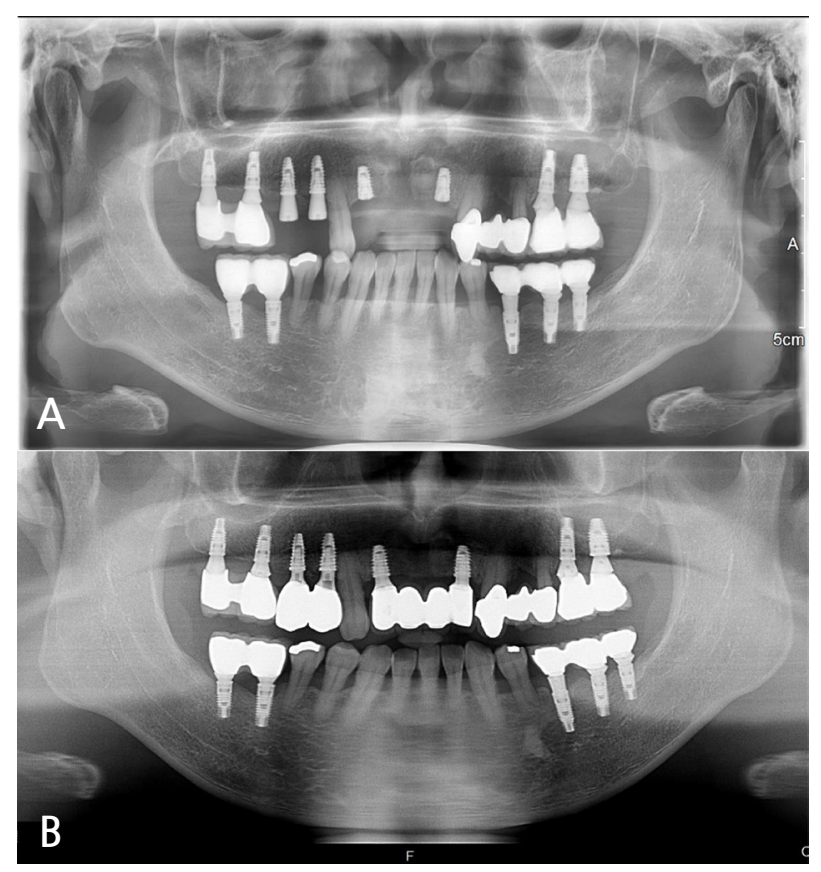

Fig. 5. Case 1: Panoramic radiograph after implant placement. (A) Postoperative panoramic radiograph, (B) panoramic radiograph at 15-month follow-up after placement of the prosthesis.

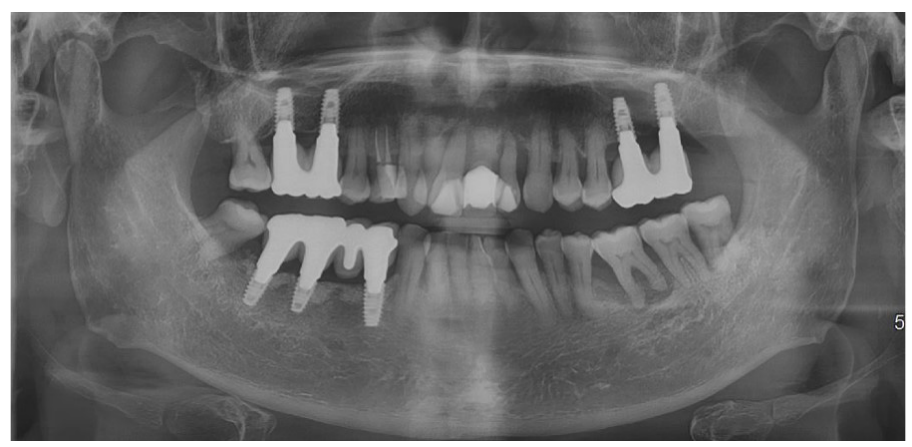

Fig. 6. Case 2: Panoramic radiograph at the first visit. 
lateral incisor area were observed. Both the areas had a concave bony morphology in the apical region (Fig. 7C, 7D). DBBM was placed in the defect and NBCM was fixed to the apical area of the remaining alveolar ridge with titanium pins. The fixed membrane was stretched enough to produce a balloon effect and fixed with titanium pins to the crestal bone (Fig. 7E, 7F). The flap was sutured with modified Laurell sutures and simple interrupted sutures using 4-0 non-resorbable nylon monofilament (Fig. 7G, 7H). The soft tissue healing pattern was presumed to be secondary healing in the right lateral incisor site after suture removal, but without any symptoms of inflammation (Fig. 8A, 8B).

After reopening for implant placement, the site demonstrated horizontal bone width of more than 7 mm after 4 months of healing (Fig. 9A, 9B). Implant placement (IS III active ${ }^{\circledR}, 4.0 \times 8.5 \mathrm{~mm}$ ) was performed without additional GBR and the flap was sutured with 4-0 non-resorbable nylon monofilament (Fig. 9C, 9D, 9E). The ISQ values of the left and right lateral incisor implants at the time

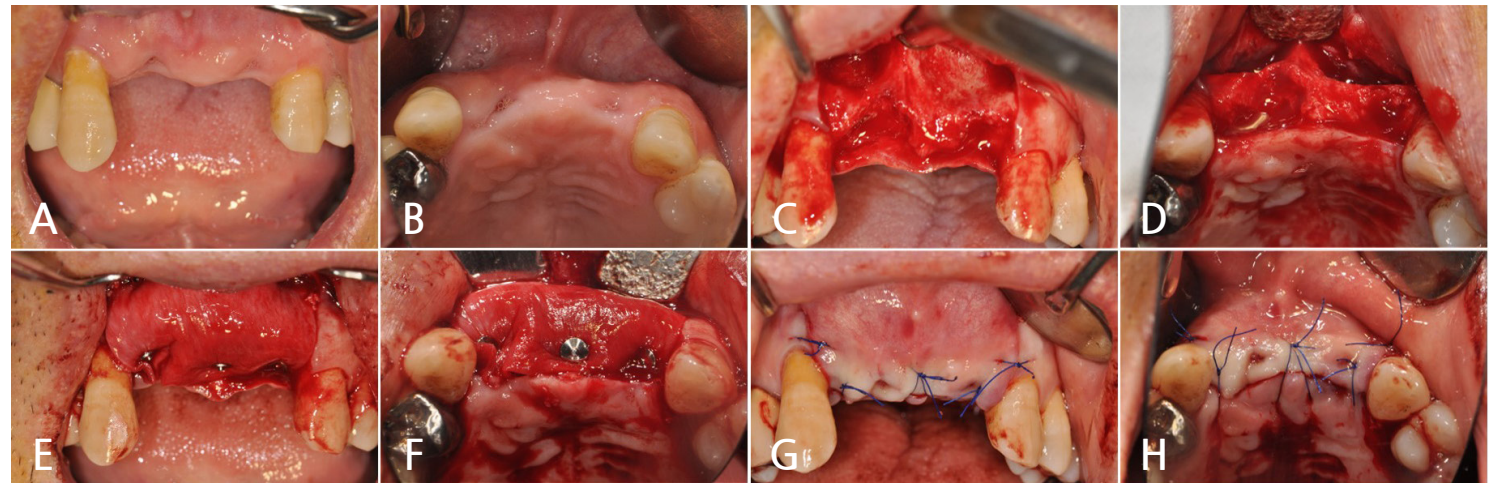

Fig. 7. Case 2: Clinical photographs of the guided bone regeneration procedure. (A), (B) Clinical situation before surgery, (C), (D) Two-wall defect in right lateral incisor area and horizontal atrophy of the alveolar bone in the left lateral incisor area observed after flap elevation, (E), (F) Deproteinized bovine bone mineral was placed in the defect and native bilayer collagen membrane was fixed with titanium pins, $(G),(H)$ Modified Laurell sutures and simple interrupted sutures were used for crestal and vertical incision lines, respectively.
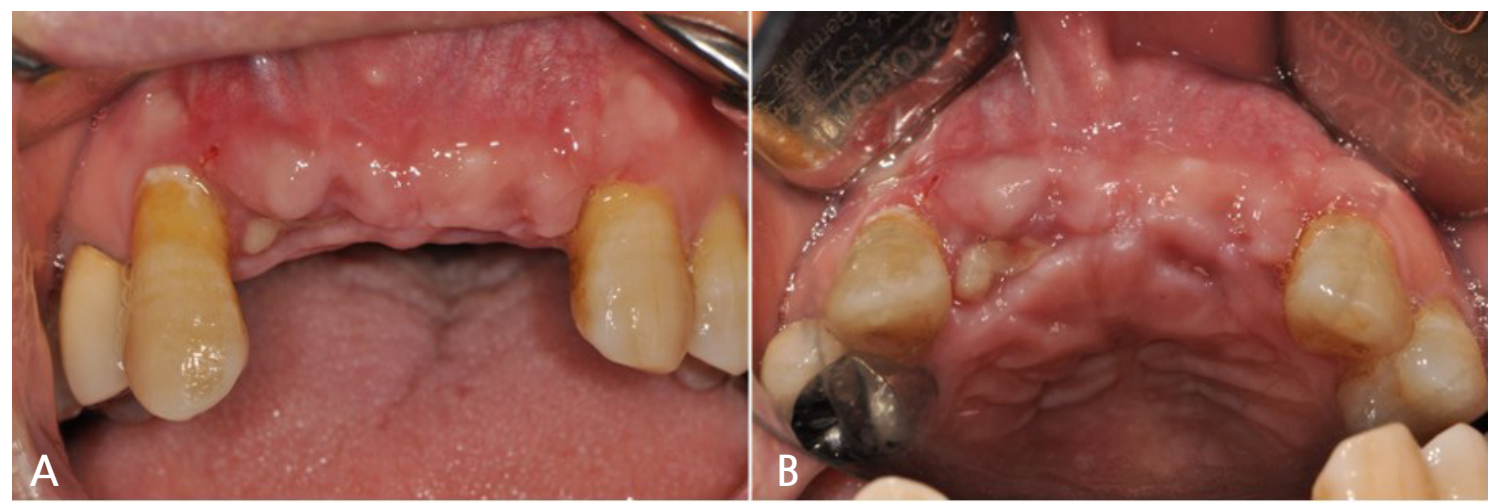

Fig. 8. Clinical photographs at suture removal. (A) Frontal view, (B) occlusal view Yellowish secondary healing pattern of the soft tissue was observed in the right lateral incisor area. 
of placement were 65 and 80 , respectively. The bone and implant conditions were maintained without any complications for 18 months after the placement of the prosthesis (Fig. 10A, 10B).

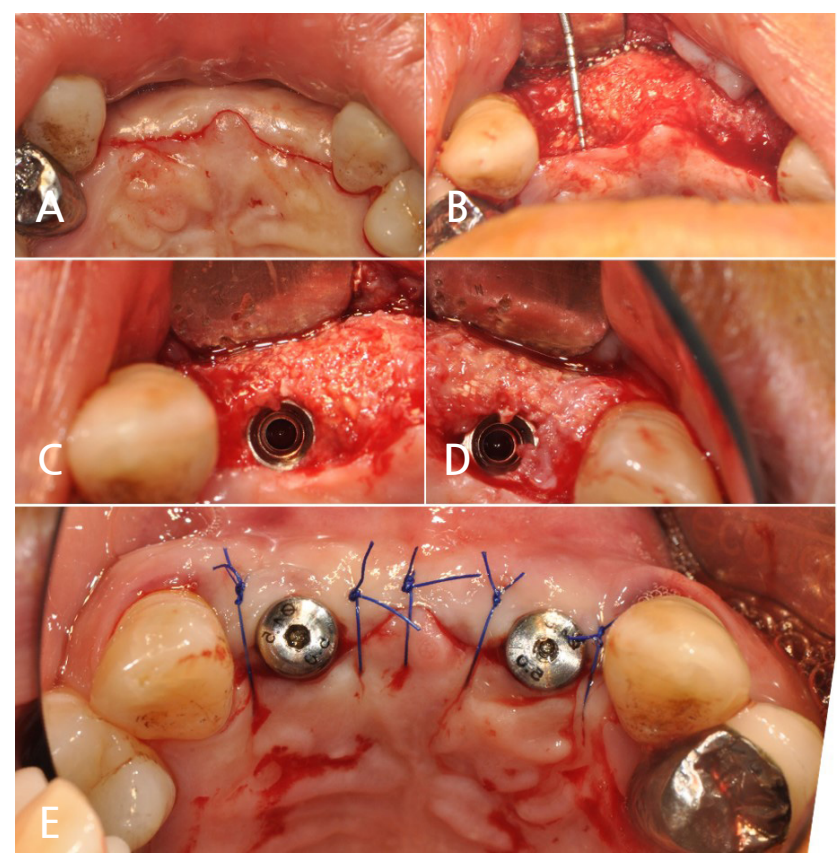

Fig. 9. Clinical photographs of implant placement. (A) Crestal incision for implant placement, (B) Horizontally augmented ridge observed after buccal flap elevation, (C), (D) Implant placement without additional guided bone regeneration, $(E)$ Simple interrupted sutures at the crestal incision.

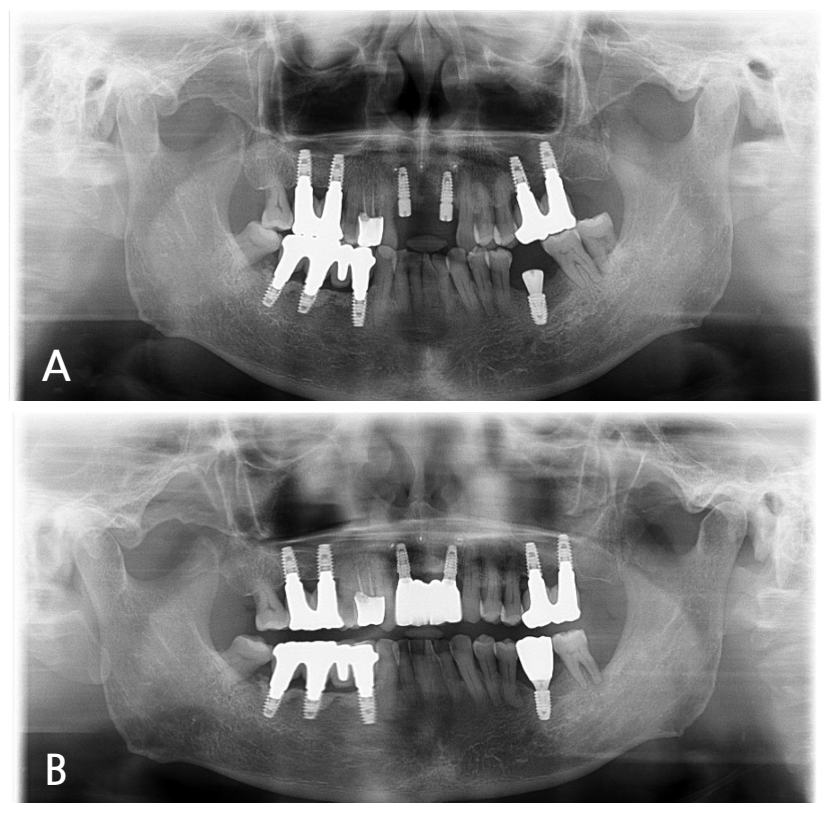

Fig. 10. Panoramic radiograph after implant placement. (A) Postoperative panoramic radiograph, (B) Panoramic radiograph at 18-month follow-up after placement of the prosthesis. 


\section{Discussion}

It is known that use of a resorbable membrane during the GBR procedure has a limited effect on the regeneration of alveolar bone due to the lack of space maintenance ability. ${ }^{6}$ The author believed that manipulation of the graft material in the crestal direction during the sausage technique would help in tight aggregation of the graft particles, leading to better space maintenance ability. Based on this theoretical estimation, it was assumed that if the graft material has sufficient strength to withstand the pressure of the resorbable membrane and pin, it can produce sufficiently good results. The author proceeded with these cases with the belief that the tightly agglomerated graft material would exhibit sufficient space maintenance ability without additional graft material manipulation. The results of this technique were acceptable.

Wound stability can be achieved through secure fixation of the membrane, which can achieve successful bone regeneration. ${ }^{8}$ In the cases from the present report, the alveolar ridge was augmented horizontally with secure wound stability using only a titanium pin and a resorbable membrane. Studies conducted by Istvan Urban have reported that natural collagen has more elasticity than cross-linked synthetic collagen and it is a reliable option to immobilize the graft. ${ }^{1}$ This mechanical property is advantageous for wound stability, which is consistent with the results of the present report. On the other hand, the spongy maxillary bone made it difficult to fix the titanium pin, threatening the wound stability. Therefore, in the maxilla, it is necessary to anatomically identify the position of the cortical bone to fix the pin for wound stability.

The soft tissue pattern after suture removal in case 2 was presumed to be secondary healing in the right lateral incisor region without any signs of inflammation. However, proper horizontal alveolar bone regeneration was achieved with no difficulty during implant placement. This was a significant result compared to the results obtained with a non-resorbable membrane, although there is a possibility of infection after membrane exposure. ${ }^{9}$ The horizontally augmented bone despite the secondary healing pattern at suture removal might imply the relative safety of a resorbable membrane even if it is exposed. In addition, the high rate of loss of grafted bone associated with the exposure of a cross-linked membrane was avoided in this case. ${ }^{2}$ It is speculated that the relatively rapid degradation of native collagen membrane is advantageous for secondary healing. ${ }^{10}$

The titanium pins at the apical area were not removed in case 2 (Fig. 9) to avoid the patient's burden regarding expanding the scope of surgery. This might result in inflammation in the distant future. However, Meloni et al. reported a high implant survival rate and high average bone augmentation without signs of inflammation at the 3-year follow-up without apical pin removal after final loading. ${ }^{11}$ 
The present report did not involve a long-term follow-up, but the potential risk seems to be low, since peri-implantitis progresses from the crestal site. Longer follow-up studies on various aspects are required in the future.

Istvan Urban recommended the use of a mixture of DBBM and autogenous bone during the sausage technique. ${ }^{1}$ Interestingly, the present cases exhibited sufficient alveolar bone regeneration for implant placement using DBBM alone. These results may be explained by the spongy nature of the maxillary anterior bone and the consequent vigorous blood supply in the area of interest. This is one of the limitations of the present report and further studies are needed to determine whether extensive horizontal cortical bone defects would exhibit similar results. In addition, it is necessary to confirm whether the sausage technique without autogenous bone graft shows stable results even when it is performed simultaneously with implant placement and whether vertical bone augmentation is possible using this technique.

\section{Conclusion}

Within the limitations of this report, the sausage technique using NBCM with DBBM was able to achieve an appropriate amount of horizontal alveolar bone augmentation for implant placement in the maxillary anterior area.

\section{References}

1. Urban I. Vertical and horizontal ridge augmentation: new perspectives. 1sted. Germany: Quintessence Publishing 2017. p. 117-46.

2. Park SH, Lee KW, Oh TJ, Misch CE, Shotwell J, Wang HL. Effect of absorbable membranes on sandwich bone augmentation. Clin Oral Implants Res 2008;19:32-41.

3. Rothamel D, Schwarz F, Sager M, Herten M, Sculean A, Becker J. Biodegradation of differently cross-linked collagen membranes: an experimental study in the rat. Clin Oral Implants Res 2005;16:369-78.

4. Tatakis DN, Promsudthi A, Wikesjo UM. Devices for periodontal regeneration. Periodontol 2000. 1999;19:59-73.

5. Kim SH, Kim DY, Kim KH, Ku Y, Rhyu IC, Lee YM. The efficacy of a double-layer collagen membrane technique for overlaying block grafts in a rabbit calvarium model. Clin Oral Implants Res 2009;20:1124-32.

6. Hämmerle C, Jung R. Bone augmentation by means of barrier membranes. Periodontol 2000. 2003;33:36-53.

7. Wang HL, Boyapati L. "PASS” principles for predictable bone regeneration. Implant Dent 2006;15:8-17. 
8. Schenk RK, Buser D, Hardwick WR, Dahlin C. Healing pattern of bone regeneration in membraneprotected defects: a histologic study in the canine mandible. Int J Oral Maxillofac Implants 1994;9:13-29.

9. Simion M, Baldoni M, Rossi P, Zaffe D. A comparative study of the effectiveness of e-PTFE membranes with and without early exposure during the healing period. Int J Periodontics Restorative Dent 1994;14:166-80.

10. Zitzmann NU, Naef R, Scharer P. Resorbable versus nonresorbable membranes in combination with Bio-Oss for guided bone regeneration. Int J Oral Maxillofac Implants 1997;12:844-52.

11. Meloni SM, Jovanovic SA, Urban I, Baldoni E, Pisano M, Tallarico M. Horizontal ridge augmentation using GBR with a native collagen membrane and 1:1 ratio of particulate xenograft and autologous bone:A3-year after final loading prospective clinical study. Clin Implant Dent RelatRes 2019;21:66977. 